\title{
Review
}

\section{Molecules controlling lymphocyte migration to the gut}

\begin{abstract}
Preface
Continuous lymphocyte migration to normal gut is a prerequisite for immune homoeostasis in humans. In this review we briefly describe the physiology of lymphocyte recirculation through the bowel. The adhesion molecules mediating the lymphocyte-endothelial interactions in the gut during the multistep extravasation cascade will be presented in the light of their ability to confer mucosal selectivity of lymphocyte trafficking. We will also discuss the relevance of leucocyte recirculation in respect to bowel inflammation and mucosal vaccination, and its potential in the anti-inflammatory treatment of gastrointestinal diseases.
\end{abstract}

\section{Lymphocyte recirculation-the general theme}

The gut is the main portal of antigen entry into the body and lymphocytes are responsible for mounting an adequate immune response against harmful antigens. As each lymphocyte only carries an antigen receptor for a single antigen, a huge number of lymphocytes, each specific for a different antigen, are produced in the bone marrow and thymus each day. These naive cells must then be able to sample freely all different tissues of the body in search of their cognate antigens. To maximise the likelyhood of the rare possibility that a given lymphocyte would find its cognate antigen introduced anywhere in the body, a sophisticated system of lymphocyte recirculation has evolved. ${ }^{1-4}$

In this process, lymphocytes continuously patrol between the blood and different tissues of the body. Initially blood borne lymphocytes leave the circulation via secondary lymphoid tissues like lymph nodes. The exit from the blood mainly takes place in distinct postcapillary vessels called high endothelial venules (HEV), which display several unique structural and functional modifications to facilitate extravasation. ${ }^{5}$ Foreign antigens are retrieved and concentrated from distal epithelial surfaces via the afferent lymphatic system into the secondary lymphoid organs.

When an extravasated lymphocyte finds its antigen in the supportive context of the secondary lymphoid tissues, it starts to proliferate and differentiate and gives rise to numerous effector cells. These progeny cells then leave the lymphoid tissues via efferent lymphatics and re-enter the systemic circulation. During this process the activated effector lymphocytes have become imprinted in a way which allows them to migrate preferentially back (home) to the sites where they became activated (the reactive secondary lymphoid organ) and to the sites from which the inciting antigen was originally drained (the challenged epithelial surfaces). At these target organs the antigen specific effector cells then effectively clear the antigenic threat. After the acute inflammation has resolved, some of these cells revert into a less activated stage and survive for years as memory cells. These cells patrol at low levels the epithelial organs all the time and afford a brisk, antigen specific secondary immune response should the same antigenic challenge recur.

\section{Lymphocyte migration into the gut}

Intestinal antigens are sampled from the gut lumen by $M$ cells which are positioned between the enterocytes. The antigens are translocated into underlying Peyer's patches (the organised secondary lymphoid organs in the gut wall) where they can be efficiently processed and introduced to lymphocytes. ${ }^{6}$ Naive lymphocytes carried by the blood enter the Peyer's patches by binding to the specialised HEV. If the lymphocyte does not find its antigen it leaves the Peyer's patch and returns into the systemic circulation via the efferent lymphatics. If, however, the $B$ or $T$ lymphocyte recognises its cognate antigen it starts to divide and differentiate. After some time the immunoblasts are transported via the lymphatics into mesenteric lymph nodes. Ultimately, the effector cells return into the blood and are randomly dispersed all over the body. When those progeny cells sooner or later re-enter the bowel vasculature they can return to the Peyer's patch via HEV. More importantly, the immunoblasts now can very efficiently bind to flat-walled venules of lamina propria and immigrate into this non-organised subepithelial lymphoid tissue of the gut, which is constantly subjected to immunostimulation. The different nature of these two lymphoid compartments is also highlighted by the fact that the molecular mechanisms directing lymphocyte migration into Peyer's patches and lamina propria are somewhat distinct although overlapping (see later).

The mucosal recirculation system opposes to that of peripheral lymph nodes (PLN) which drains antigens mainly from the skin. The whole concept of tissue selective lymphocyte homing was, in fact, worked out in the 1960s and 1970s by following the fate of transferred mucosal immunoblasts. ${ }^{8-11}$ In those in vivo animal experiments, gut derived immunoblasts showed 5-20-fold better localisation back to bowel than to PLN. It is traditionally thought that oral, intestinal, respiratory, mammary, and urogenital surfaces form a common mucosal lymphatic system which also constitutes a functionally unified recirculation system. ${ }^{12}$ More recently this view has been challenged. ${ }^{13}$ Furthermore, there are clearly some interconnections between the gut and peripheral tissues like joint synovium and pancreas as gut derived lymphocytes have a propensity for selective homing to these organs ${ }^{14}{ }^{15}$ when compared with PLN or skin.

\section{Multistep nature of the extravasation cascade}

Lymphocyte adhesion to vascular endothelium is much more than just a simple juxtaposition of two stationary cells. The shear force introduced by the blood flow and the rapid change of a lymphocyte from a non-adhesive blood cell into an adhesive cell in the extravascular compartment necessitates a complex cascade of successive but separable phases of cell-cell interaction. ${ }^{16}{ }^{17}$

Initially, the blood borne lymphocyte is displaced from the central flow of the vessel and collides with the vascular endothelium in HEV (fig 1). ${ }^{19}$ These tethering contacts

Abbreviations used in this article: HEV, high endothelial venules; PLN, peripheral lymph nodes; MAdCAM, mucosal addressin cell adhesion molecule; PNAd, peripheral lymph node addressins; EGF, epidermal growth factor; ICAM, intercellular adhesion molecule; SLC, secondary lymphoid-tissue chemokine; TNF, tumour necrosis factor; VAP, vascular adhesion protein; LFA, lymphocyte function associated antigen. 




Figure 1 Multistep model of lymphocyte homing into the gut. The five separate steps in lymphocyte trafficking from the blood into gut are shown. The molecules mediating different stages of binding of naive lymphocytes to Peyer's patch (PP) high endothelial venules (HEV) and adhesion of activated immunoblasts to Peyer's patch HEV or flat walled venules in lamina propria are shown. In the three first steps only molecules which have been shown to function in vivo under flow conditions in the gut are included. MAdCAM, mucosal addressin cell adhesion molecule; ICAM, intercellular adhesion molecule; LFA, lymphocyte function associated antigen; MAdCAMcho, MAdCAM with special carbohydrate structures.

lead the cells to adopt a rolling behaviour-that is, they start to roll down the vascular lining at a velocity (10-50 $\mu \mathrm{m} / \mathrm{s}$ ) considerably slower than that of erythrocytes (typically $>4000 \mu \mathrm{m} / \mathrm{s}$ ). If the rolling cell locally receives appropriate triggering signals from the tissue, the lymphocyte can become activated. Then the cell can firmly bind to the endothelium, resist the shear stress and actually halt in the vessel. The stably bound cell finally seeks interendothelial junctions, and penetrates through them and the underlying basement membrane into the tissue. There it perceives other directional signals which help it to move within the tissue stroma.

Lymphocyte binding to endothelial cells involves adhesion molecules expressed on the surface of the two opposing cells types. ${ }^{1-3} 20$ During the multistep cascade molecules from many adhesion molecule superfamilies are successively brought into play (figs 1 and 2). The rapid and readily reversible contacts moderating tethering and rolling are mediated by selectins and their glycoprotein counterparts. ${ }^{21}$ These molecules are constitutively active and are presented on the tip of microvillous projections well above the planar cell surface, which renders them highly suitable for making initial contacts. The activation step is generally thought to involve binding of chemoattractants and other chemotactic cytokines secreted or presented by the endothelium to their seven-pass transmembrane receptors on lymphocytes. ${ }^{22}$ The ensuing signalling cascade triggers a change in the affinity and/or avidity of constitutively expressed inactive integrin molecules on the lymphocytes. ${ }^{23}$ The interaction of the activated integrins with their endothelial ligands, mainly belonging into a immunoglobulin superfamily, ultimately secures the contacts between the lymphocyte and the vessel wall. The molecular basis of the transmigration is poorly understood, ${ }^{24}$ but it may involve some of the same molecules that are used for stable arrest together with directional clues from chemoattractants and the cellular machinery that permits cell movement and penetration through the matrix.

The selectivity of the migration is determined by combinations of decisions at different steps of the emigration cascade. ${ }^{17}$ Thus, there are homing receptors relatively specifically expressed on gut seeking lymphocyte subpopulations and certain endothelial addressins are also almost exclusively synthesised in the intestine. Moreover, gut specific chemokines are likely to be found, although none of the 50 species characterised so far has been directly shown to function as a bowel specific leucocyte activator and chemoattractant. Firm adhesion and the later steps in the cascade are mediated by molecules which display little tissue selectivity as such, but nevertheless are absolutely required for a successful emigration cascade to be executed. The gut also serves as an example of a site that has been particularly

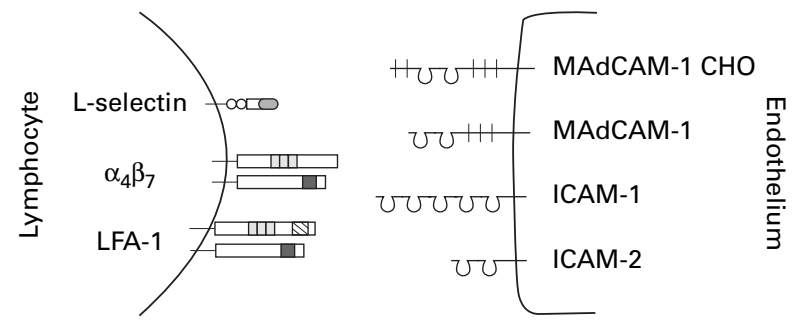

\begin{tabular}{|rll|}
\hline$\square$ & Selectin & Hucin \\
0 & Lectin & M Immunoglobulin \\
$\square$ & EGF-like & \\
0 & CR repeat & \\
$\square$ & Integrin \\
$\square$ & Metal binding \\
$\square$ & I-domain \\
$\square$ & I-domain-like
\end{tabular}

Figure 2 Structure of the mucosal adhesion antigens. The mosaic nature of L-selectin, mucosal addressin cell adhesion molecule (MAdCAM) 1 and special carbohydrate containing forms of MAdCAM-1 (MAdCAM-1 $\mathrm{CHO}$ ) is shown together with the prototypic structure of integrins and intercellular adhesion molecules (ICAM). Lectin, C-type (calcium dependent) lectin domain; EGF-like, epidermal growth factor-like domain; and $C R$, complement regulatory protein-like motif in selectins. Metal binding = divalent cation binding repeats in a subunits of integrins; I-domain $=$ inserted domain in aL subunit of lymphocyte function associated antigen (LFA) 1 and I-domain-like, a putative inserted domain-like area in $\beta$ subunits of integrins. 
thoroughly studied in respect of the overlapping function of distinct adhesion molecules (see later)..$^{18} 25$

Next we will present those adhesion molecules which are most relevant to lymphocyte homing into the gut. The description of the structure and function of many other molecules involved in leucocyte-endothelial adhesion may be found in recent review articles and in the original references therein..$^{1-3} 20$

\section{Molecular control of lymphocyte traffic in mucosal tissues under physiological conditions \\ LYMPHOCYTE RECEPTORS \\ L-selectin}

Naive cells enter the mucosal sites via organised mucosal lymphoid tissues. They express high levels of L-selectin (CD62L), the principal homing receptor for PLN, and use it for tethering to mucosal endothelium. L-selectin interacts with a special form of mucosal addressin cell adhesion molecule-1 (MAdCAM-1) that is adorned with carbohydrate epitopes normally present in peripheral lymph node addressins (PNAd). ${ }^{26}$ These carbohydrate moieties function as ligands for L-selectin. The importance of L-selectin in mucosal homing of naive lymphocytes has been shown in studies in which anti-L-selectin antibody treatment partially inhibits homing of lymphocytes to Peyer's patches. ${ }^{27}$ Moreover, L-selectin knockout mice show diminished trafficking of lymphocytes to Peyer's patches, ${ }^{28}$ thus confirming the conclusions based on the antibody treatment studies.

L-selectin belongs to the family of selectins that all characteristically have a lectin domain, an epidermal growth factor (EGF) like area and variable numbers of repeats similar to those found in complement regulatory proteins (fig 2). The lectin domain is the most central structure of the molecule in ligand binding because it interacts efficiently with molecules containing certain sialylated, fucosylated, and sulfated carbohydrate structures. ${ }^{21}$

\section{$\alpha_{4} \beta_{7}$}

Unlike naive lymphocytes, gut homing blasts and memory cells express very low levels of L-selectin or lack it completely. ${ }^{29}$ They do not need L-selectin to home to Peyer's patches or the appendix. ${ }^{30}$ Instead, as a result of their maturation stage they can skip the selectin mediated phase in the multistep adhesion cascade and bind directly to endothelium via an activated integrin, $\alpha_{4} \beta_{7}{ }^{18} \alpha_{4} \beta_{7}$ is also the key receptor for the entrance of lymphocytes into the lamina propria. ${ }^{18}$ Interestingly, $\alpha_{4} \beta_{7}$, the principal mucosal homing receptor, functions at several steps of the adhesion cascade by interacting with MAdCAM $-1 .^{25}$ It is able to act as a rolling receptor as well as a receptor mediating firm binding to endothelium. The central role of $\alpha_{4} \beta_{7}$ in mucosal homing is unequivocally illustrated by the gene targeted mice lacking $\alpha_{4} \beta_{7}$. In these animals, formation of mucosa associated lymphatic tissues is severly impaired and $\beta 7$ negative lymphocytes fail to adhere to Peyer's patch HEV. ${ }^{31}$

$\alpha_{4} \beta_{7}$ is a member of the large family of integrins. As all integrins, it is a heterodimeric molecule consisting of noncovalently associated $\alpha$ and $\beta$ chains. Naive lymphocytes express low levels of $\alpha_{4} \beta_{7}$ but upon activation a significant amount of $\alpha_{4} \beta_{7}$ appears on the lymphocyte surface in a functionally active form. ${ }^{32}$

\section{Lymphocyte function associated antigen}

Naive lymphocytes and most likely also immunoblasts and memory cells utilise lymphocyte function associated antigen (LFA) 1 (CD11a/CD18, $\alpha \mathrm{L} \beta 2)$ in the later steps of the adhesion cascade to arrest and transmigrate into mucosal sites. ${ }^{25}$ LFA-1 is a multifunctional adhesion molecule involved in various adhesive interactions in the immune system including antigen presentation and $\mathrm{T}$ cell mediated killing. ${ }^{33}$ Patients completely lacking LFA-1 and other $\beta 2$ integrins die at an early age because their granulocytes are not able to extravasate to sites of inflammation. ${ }^{34}$ However, lymphocyte homing is not severely impaired indicating that other molecules, especially $\alpha 4$ integrins, can compensate for the lack of LFA-1 in lymphocyte homing.

LFA- 1 is a $\beta 2$ integrin which are also known as leucocyte integrins. It shares the $\beta$ chain with three other members of the leucocyte integrin family. LFA-1 needs to be either in the high affinity stage or clustered to interact efficiently with its endothelial cell ligands, intercellular adhesion molecules (ICAMs), via its inserted domain (I domain). ${ }^{23}$

$\alpha E \beta_{7}$

It is obvious that following the transmigration step various intercellular and cell-matrix interactions occur when the lymphocyte travels and finds its final destination within the mucosa associated lymphatic tissues. One molecule thought be important in determining the destination of intraepithelial lymphocytes is the $\alpha \mathrm{E} \beta_{7}$ integrin. It is prominently expressed on intraepithelial lymphocytes and is able to use E-cadherin present on epithelial cells as its ligand. ${ }^{35}$ Thus, this receptor-ligand pair may be important in retaining the intraepithelial lymphocytes in their anatomical location.

\section{Other molecules}

CD44 is a multifunctional adhesion proteoglycan molecule able to mediate lymphocyte rolling on hyaluronate and to activate LFA-1.36 ${ }^{37}$ Lymphocyte binding to mucosal endothelium can be partially blocked by anti-CD44 antibodies in vitro. ${ }^{38}$ As CD 44 deficient mice have not been reported to have abnormalities in their mucosa associated lymphatic tissues, ${ }^{39}$ the role of CD 44 may not, however, be central in the homing of lymphocytes to normal mucosal sites.

In vivo inhibition studies have shown that blockade of the sialomucin CD43 results in diminished homing of lymphocytes into several organs including Peyer's patches. ${ }^{40}$ In contrast, knockout animals show increased homing capacity of lymphocytes indicating that CD43 may regulate the function of other homing associated molecules. ${ }^{41}$

\section{ENDOTHELIAL LIGANDS FOR LYMPHOCYTE RECEPTORS}

$M A d C A M-1$

MAdCAM-1 is preferentially expressed on vascular endothelium at mucosal sites both in organised lymphoid tissues and in the lamina propria. ${ }^{42}$ In flat walled vessels in lamina propria MAdCAM-1 is not decked with carbohydrates functioning as L-selectin ligands and therefore in these vessels it mediates the entrance of blasts and memory cells, namely the cells in which $\alpha_{4} \beta_{7}$ has already been activated. ${ }^{18}$ The importance of MAdCAM-1 in lymphocyte homing to mucosal sites is clearly shown by the significantly reduced homing of lymphocytes to mucosa associated lymphatic tissues after the treatment with anti-MAdCAM-1 antibody. ${ }^{43}$ Moreover, long term antibody treatment results in severe hypoplasia of Peyer's patches. ${ }^{14}$

MAdCAM-1 belongs to the immunoglobulin (Ig) superfamily but has also a mucin-like domain that is heavily $O$-glycosylated (fig 2 ). The Ig domains are crucial for binding to $\alpha_{4} \beta_{7}$, whereas the mucin-like structure has been thought to act as a stalk facilitating the presentation of the Ig domains to $\alpha_{4} \beta_{7}{ }^{44}$ 
ICAMs

ICAM-1 (CD54) and ICAM-2 (CD102), members of the immunoglobulin superfamily (fig 2 ), are widely present in vasculature throughout the body. In the context of mucosal homing, they act as LFA-1 ligands mediating later steps in the extravasation cascade..$^{25}$ The most N-terminal domain of ICAM-1 and - 2 is the counter-structure for LFA-1, and dimerisation of ICAM-1 is reported to be important for the functional activity of the molecule. ${ }^{45}$

\section{CHEMOKINES}

Chemokines are thought to be required to trigger a rolling lymphocyte to become an adherent one by activating the integrins. However, most chemokines that are capable of attracting lymphocytes most likely exert their action within lymphoid tissues and thus are central in determining the travel routes and final destination of the lymphocytes within the organ. In contrast, secondary lymphoid-tissue chemokine (SLC) (also known as 6Ckine, Exodus-2, and thymus derived chemotactic agent 4) is strategically located in HEV both in Peyer's patches and PLN. ${ }^{46}$ SLC increases $\alpha_{4} \beta_{7}$ mediated rolling and firm arrest on recombinant MAdCAM-1 in vitro. ${ }^{47}$ Moreover, SLC augments LFA-1 mediated attachment of lymphocytes to ICAM-1. ${ }^{46}$ SLC is also expressed in $T$ cell areas in the lymph nodes and may thus additionally help to guide lymphocytes within the lymphoid tissues. ${ }^{46}$

Chemokines are small soluble heparin binding proteins (excluding fractalkine which is larger and also has a transmembrane form) that function as chemoattractants for different subsets of leucocytes. ${ }^{22}$ They can be divided into four families that are defined by a cysteine signature motif: CXC (e.g. interleukin-8), CC (e.g. SLC), C (lymphotactin and $\mathrm{CX} 3 \mathrm{C}$ (fractalkine)), where $\mathrm{C}$ is a cysteine and $\mathrm{X}$ any amino acid residue. Seven transmembrane domain molecules coupled to G-proteins serve as their ligands. Ligand binding leads to modulation of the activation of intracellular signal transduction enzymes (adenylate cyclase and phospholipase C) with subsequent signalling effects. Proteoglycan molecules are thought to present soluble chemokines to lymphocytes at the endothelial cell surface. ${ }^{22} 48$

\section{Inflammation induced changes in the traffic of mucosal lymphocytes}

During inflammation the same principles apply to the extravasation cascade as during physiological recirculation. ${ }^{49}$ Together with the haemodynamic changes the net effect of inflammatory activation of endothelial cells and leucocytes is an increased influx of both lymphocytes and granulocytes to sites of inflammation. Expression of existing endothelial cell molecules such as MAdCAM-1 and ICAM-1 is upregulated, and some inflammation inducible molecules such as E-selectin (CD62E) and P-selectin (CD62P), practically absent under normal circumstances, appear on the endothelium. ${ }^{29} 4250$ Kinetic studies on the expression of ICAM-1 and E- and P-selectin in mouse small intestine have been performed after experimental administration of cytokines and endotoxin. Tumour necrosis factor (TNF) $\alpha$ causes upregulation of ICAM-1 that reaches its maximum by six hours. Relatively rapid $(<1 \mathrm{~h})$ induction of $\mathrm{P}$-selectin can be caused by histamine and more prolonged expression of P-selectin can be induced by endotoxin. Endotoxin induced E-selectin expression is maximal at three hours and thereafter it rapidly disappears from the endothelial cell surface in gut. ${ }^{50} \mathrm{E}-$ and P-selectin, instead of controlling lymphocyte entrance may, however, rather facilitate the extravasation of granulocytes to inflammatory foci.
Vascular adhesion protein-1 (VAP-1) is one of those molecules that is expressed in low levels in normal intestine but is translocated from the intracellular granules to the endothelial cell surface upon elicition of inflammation. Significant induction of PNAd, which normally mediates lymphocyte traffic to PLN, also takes place during gut inflammation. PNAd together with VAP-1 also allow the entrance of the lymphocytes belonging to the PLN recirculatory pool to inflamed mucosal sites. ${ }^{29}{ }^{51}$ Aberrant homing of these cells to mucosal sites may be a central element in the pathogenesis of inflammatory bowel disease (IBD), because these cells most likely actively participate in the process resulting in tissue damage.

\section{Mucosal recirculation and vaccine development}

Organ specific lymphocyte trafficking routes have direct implications for the development of new vaccines, especially in connection to selection of the site of vaccination. As lymphocytes activated in a certain organ system tend to home back to sites of their activation, immunisation protocols that evoke the response in a subset of lymphocytes bearing high levels of mucosal homing receptor, $\alpha_{4} \beta_{7}$, will presumably provide the best protection against mucosal pathogens. The importance of the vaccination site to the nature of the immune response has been confirmed experimentally by vaccinating volunteers orally or parenterally with the same antigen. The antigen given orally induces significantly more antibody producing cells with a $\alpha_{4} \beta_{7}$ phenotype than the same antigen given parenterally. ${ }^{52}$ In an analogous way, $T$ cells activated via mucosal routes tend to bear more $\alpha_{4} \beta_{7}$ than those activated systemically. ${ }^{54}$

An exciting possibility is the regulation of the expression of homing associated molecules of the effector cell populations by cytokines and other factors or drugs, and thus redirect the homing of the protection providing lymphocyte populations in the body to sites where the elimination of a pathogen is needed. However, much basic and applied work needs to be performed to elucidate the feasibility of this approach.

\section{Therapeutic intervention in mucosal homing}

As the inflammatory component is a major feature in a vast number of gastrointestinal diseases, the advent of molecular understanding of leucocyte trafficking in mucosa has fuelled enthusiasm for the development of novel antiinflammatory strategies. The basic idea is that, regardless of the aetiology, excessive, prolonged or otherwise inappropriate gut inflammation can be terminated by blunting the multistep adhesion cascade at any step. Most studies testing this concept have used mouse monoclonal antibodies or their humanised versions, but in future receptor or ligand analogs and small molecular drugs are the likely formulas to be used in clinics.

Inflammatory bowel disease has been succesfully targeted using anti-adhesion therapy. In initial trials anti- $\alpha 4$ monoclonal antibodies (recognizing both $\alpha_{4} \beta_{1}$ and $\alpha_{4} \beta_{7}$ ) noticeably relieved gut inflammation in a cotton-top tamarin model. ${ }^{55}$ An antibody specific for the principal mucosal homing receptor, $\alpha_{4} \beta_{7}$, has also been used in the same model. In these chronically colitic animals the stool consistency improved within a day, the number of cells in the inflammatory infiltrate decreased after intravenous treatment with the antibody and the favourable effects were long lasting. ${ }^{56}$ Similar findings have been produced in a mouse model of IBD, both after blocking $\alpha_{4} \beta_{7}$ and after targeting MAdCAM-1. ${ }^{57}$ In another approach, antisense oligonucleotides preventing synthesis of ICAM-1 were administred to patients with IBD. ${ }^{58}$ Although the number 
of patients enrolled in the study was small, the authors concluded that the treatment was safe. However, it did not seem to alleviate significantly most symptoms and signs of the disease.

Adhesion molecules also play a central role in the pathogenesis of radiation enteropathy. Blocking of LFA-1 and ICAM-1 has been shown to be benefical in treating this postirradiation syndrome in rats. ${ }^{59}$ Ischemia-reperfusion injury of the gut is also notably attenuated if the function of P-selectin is neutralised with monoclonal antibodies. ${ }^{60}$ The leucocytic infiltration caused by Clostridium difficile toxin A can also be abrogated to a large extent if the function of the leucocyte-endothelial adhesion molecules (e.g. P-selectin and LFA-1), induced indirectly by this toxin, is blocked. ${ }^{61}$ These examples have been mentioned just to illustrate the concept that anti-adhesive therapy can be a broad spectrum and at the same time a precise anti-inflammatory device, only affecting the involved gut area if the target molecules are selected appropriately. Suprisingly, very few, if any, side effects have been reported in these trials. This suggests that short term interference in mucosal recirculation can be well tolerated without jeopardising the vital need for mucosal immune homoeostasis.

\section{Concluding remarks}

Molecular understanding of the mechanisms which guide lymphocyte trafficking into the gut is rapidly increasing. At the moment we know that in this anatomical compartment lymphocyte $\alpha_{4} \beta_{7}$ and endothelial MAdCAM- 1 are the most critical elements, which nevertheless need the help of other molecules for successful extravasation to occur. Changes in lymphocyte homing into the gut may cast light on the pathogenesis of intestinal inflammation. Moreover, there are realistic expectations that molecular dissection of this recirculation pathway will help to design rational vaccination regimens and new anti-inflammatory strategies.

M SALMI S JALKANEN

MediCity Research Laboratory,

University of Turku and National Public

Health Institute Department in Turku,

Finland

Correspondence to: Dr S Jalkanen, MediCity Research Laboratory,

Tykistökatu 6A, FIN-20520 Turku, Finland (email: sirpa.jalkanen@utu.fi).

1 Springer TA. Traffic signals for lymphocyte recirculation and leukocyte emigration: the multistep paradigm. Cell 1994;76:301-14

2 Butcher EC, Picker LJ. Lymphocyte homing and homeostasis. Science 1996; 272:60-6.

3 Salmi M, Jalkanen S. How do lymphocytes know where to go: current concepts and enigmas of lymphocyte homing. Adv Immunol 1997;64:139-218 4 Imhof BA, Dunon D. Leukocyte migration and adhesion. Adv Immunol 1995; $58: 345-416$

5 Girard J-P, Springer TA. High endothelial venules (HEVs): specialized endothelium for lymphocyte migration. Immunol Today 1995;16:449-57.

6 MacDonald TT. Effector and regulatory lymphoid cells and cytokines in mucosal sites. Curr Top Microbiol Immunol 1999;236:113-35.

7 Butcher EC. Lymphocyte homing and intestinal immunity. In: Ogra PL, Lamm ME, Bienenstock J, et al, eds. Mucosal immunology. 2nd edn. Academic Press, 1999:507-22.

8 Gowans JL, Knight EJ. The route of re-circulation of lymphocytes in rat. Proc $R$ Soc Lond Ser B 1964;159:257-82.

9 Guy-Grand D, Griscelli C, Vassalli P. The gut-associated lymphoid system: nature and properties of the large dividing cells. Eur $\mathcal{f}$ Immunol 1974;4:435-43.

10 Hall JG, Hopkins J, Orlans E. Studies on the lymphocytes of sheep. III. Destination of lymph-borne immunoblasts in relation to their issue of origin. Eur F Immunol 1979;7:30-7.

11 Rose ML, Parrott DMV, Bruce RG. Migration of lymphoblasts to the small intestine. II. Divergent migration of mesenteric and peripheral immunoblasts to sites of inflammation in the mouse. Cell Immunol 1976;27:36-46.

12 McDermott MR, Bienenstock J. Evidence for a common mucosal immunologic system. I. Migration of B immunoblasts into intestinal, respiratory, and genital tissues. F Immunol 1979;122:1892-8.

13 Brandtzaeg P. Mucosal immunity in the female genital tract. $\mathcal{F}$ Reprod Immunol 1997;36:23-50.

14 Hänninen A, Jaakkola I, Jalkanen S. Mucosal addressin is required for the development of diabetes in nonobese diabetic mice. F Immunol 1998;160; $6018-25$.

15 Salmi M, Rajala P, Jalkanen S. Homing of mucosal leukocytes to joints. Distinct endothelial ligands in synovium mediate leukocyte-subtype specific adhesion. $\mathcal{F}$ Clin Invest 1997;99:2165-72.
16 Spangrude GJ, Braaten BA, Daynes RA. Molecular mechanisms of lymphocyte extravasation. I Studies of two selective inhibitors of lymphocyte extravasation. I. Studies of two selec
lymphocyte recirculation. $\mathcal{F}$ Immunol $1984 ; 132: 354-62$.

17 Butcher EC. Leukocyte-endothelial cell recognition: three (or more) steps to specificity and diversity. Cell 1991;67:1033-6.

18 Berlin C, Bargatze RF, Campbell JJ, et al. a4 integrins mediate lymphocyte attachment and rolling under physiologic flow. Cell 1995;80:413-22.

19 Warnock RA, Askari S, Butcher EC, et al. Molecular mechanisms of lymphocyte homing to peripheral lymph nodes. $\mathcal{f}$ Exp Med 1998;187:20516.

20 Carlos TM, Harlan JM. Leukocyte-endothelial adhesion molecules. Blood 1994;84:2068-101.

21 Kansas GS. Selectins and their ligands: current concepts and controversies. Blood 1996;88:3259-87.

22 Rollins BJ. Chemokines. Blood 1997;90:909-28.

23 Diamond MS, Springer TA. The dynamic regulation of integrin adhesiveness. Curr Biol 1994;4:506-17.

24 Bianchi E, Bender JR, Blasi F, et al. Through and beyond the wall: late steps in leukocyte transendothelial migration. Immunol Today 1997;18:586-91.

25 Bargatze RF, Jutila MA, Butcher EC. Distinct roles of L-selectin and integrins $\alpha_{4} \beta_{7}$ and LFA-1 in lymphocyte homing to Peyer's patch-HEV in situ: the multistep model confirmed and refined. Immunity 1995;3:99108.

26 Berg EL, McEvoy LM, Berlin C, et al. L-selectin-mediated lymphocyte rolling on MAdCAM-1. Nature 1993;366:695-8.

27 Hamann A, Jablonski-Westrich D, Jonas P, et al. Homing receptors reexamined: mouse LECAM-1 (MEL-14 antigen) is involved in lymphocyte migration into gut-associated lymphoid tissue. Eur f Immunol 1991;21:2925-9.

28 Arbonés ML, Ord DC, Ley K, et al. Lymphocyte homing and leukocyte rolling and migration are impaired in L-selectin-deficient mice. Immunity 1994;1:247-60.

29 Salmi M, Granfors K, MacDermott R, et al. Aberrant binding of lamina propria lymphocytes to vascular endothelium in inflammatory bowel propria lymphocytes to vascular endothel.

30 Hamann A, Andrew DP, Jablonski-Westrich D, et al. Role of $\alpha_{4}$-integrins in lymphocyte homing to mucosal tissues in vivo. F Immunol 1994;152:328293.

31 Wagner N, Löhler J, Kunkel EJ, et al. Critical role for $\beta 7$ integrins in formation of the gut-associated lymphoid tissue. Nature 1996;382:366-70.

32 Erle DJ, Briskin MJ, Butcher EC, et al. Expression and function of the MAdCAM-1 receptor, integrin $\alpha 4 \beta 7$, on human leukocytes. F Immunol 1994;153:517-28.

33 Stewart M, Thiel M, Hogg N. Leukocyte integrins. Curr Opin Cell Biol 1995;7:690-6.

34 Etzioni A. Adhesion molecules-their role in health and disease. Pediatr Res 1996;39:191-8.

35 Cepek KL, Shaw SK, Parker CM, et al. Adhesion between epithelial cells and $\mathrm{T}$ lymphocytes mediated by E-cadherin and the $\alpha_{\mathrm{E}} \beta_{7}$ integrin. Nature 1994:372:190-3.

36 Koopman G, van Kooyk Y, de Graaff M, et al. Triggering of the CD44 antigen on T lymphocytes promotes T cell adhesion through the LFA-1 pathway. F Immunol 1990;1 145:3589-93.

37 DeGrendele HC, Estess P, Picker LJ, et al. CD44 and its ligand hyaluronate mediate rolling under physiologic flow: a novel lymphocyte-endothelial cell primary adhesion pathway. $\mathcal{F}$ Exp Med 1996;183:1119-30.

38 Jalkanen S, Bargatze RF, de los Toyos J, et al. Lymphocyte recognition of high endothelium: antibodies to distinct epitopes of an $85-95-\mathrm{kD}$ glycoprotein antigen differentially inhibit lymphocyte binding to lymph node, mucosal, or synovial endothelial cells. F Cell Biol 1987;105:983-90.

39 Schmits R, Filmus J, Gerwin N, et al. CD44 regulates hematopoietic progenitor distribution, granuloma formation, and tumorigenicity. Blood 1997;90:2217-33.

40 McEvoy LM, Sun H, Frelinger JG, et al. Anti-CD43 inhibition of T cell homing. F Exp Med 1997; 185:1493-8.

41 Stockton BM, Cheng G, Manjunath N, et al. Negative regulation of T cell homing by CD43. Immunity 1998;8:373-81.

42 Briskin M, Winsor-Hines D, Shyjan A, et al. Human mucosal addressin cell adhesion molecule-1 is preferentially expressed in intestinal tract and associated lymphoid tissue. Am ₹ Pathol 1997;151:97-110.

43 Streeter PR, Berg EL, Rouse BTN, et al. A tissue-specific endothelial cell molecule involved in lymphocyte homing. Nature 1988;331:41-6.

44 Tan K, Casasnovas JM, Liu JH, et al. The structure of immunoglobulin superfamily domains 1 and 2 of MAdCAM-1 reveals novel features important for integrin recognition. Structure 1998;6:793-801.

45 Gahmberg CG. Leukocyte adhesion: CD11/CD18 integrins and intercellular adhesion molecules. Curr Opin Cell Biol 1997;9:643-650.

46 Gunn MD, Tangemann K, Tam C, et al. A chemokine expressed in lymphoid high endothelial venules promotes the adhesion and chemotaxis of naive T lymphocytes. Proc Natl Acad Sci USA 1998;95:258-63.

47 Pachynski RK, Wu SW, Gunn MD, et al. Secondary lymphoid-tissue chemokine (SLC) stimulates integrin $\alpha_{4} \beta_{7}$-mediated adhesion of lymphocytes to mucosal addressin cell adhesion molecule-1 (MAdCAM-1) under flow. $\mathcal{F}$ Immunol 1998;161:952-6.

48 Schall TJ, Bacon KB. Chemokines, leukocyte trafficking, and inflammation. Curr Opin Immunol 1994;6:865-73.

49 Cines DB, Pollak ES, Buck CA, et al. Endothelial cells in physiology and in the pathophysiology of vascular disorders. Blood 1998;91:3527-61

50 Panés J, Granger DN. Leukocyte-endothelial cell interactions: molecular mechanisms and implications in gastrointestinal disease. Gastroenterology 1998;114:1066-90

51 Salmi M, Kalimo K, Jalkanen S. Induction and function of vascular adhesion protein-1 at sites of inflammation. F Exp Med 1993;178:225560.

52 Kantele A, Kantele JM, Savilahti E, et al. Homing potentials of circulating lymphocytes in humans depend on the site of activation. Oral, but not parenteral, typhoid vaccination induces circulating antibody-secreting cells that all bear homing receptors directing them to the gut. F Immunol 1997; 158:574-9.

53 Quiding-Järbrink M, Nordström I, Granström G, et al. Differential expression of tissue-specific adhesion molecules on human circulating antibodyforming cells after systemic, enteric, and nasal immunizations. A molecular basis for the compartmentalization of effector B cell responses. 7 Clin Invest 1997;99:1281-6. 
54 Rott LS, Rose JR, Bass D, et al. Expression of mucosal homing receptor $\alpha_{4} \beta_{7}$ by circulating $\mathrm{CD} 4^{+}$cells with memory for intestinal rotavirus. 7 Clin Invest

1997;100:1204-8.
Podolsky DK, Lobb R, King N, et al. Attenuation of colitis in the Cotton-top tamarin by anti- $\alpha 4$ integrin monoclonal antibody. F Clin Invest 1993;92: $372-80$.

56 Hesterberg PE, Winsor-Hines D, Briskin MJ, et al. Rapid resolution of chronic colitis in the cotton-top tamarin with an antibody to a gut-homing integrin alpha 4 beta 7. Gastroenterology 1996;111:1373-80.

57 Picarella D, Hurlbut P, Rottman J, et al. Monoclonal antibodies specific for $\beta_{7}$ integrin and mucosal addressin cell adhesion molecule-1 (MAdCAM-1) reduce inflammation in the colon of scid mice reconstituted with
CD45RB ${ }^{\text {high }} \mathrm{CD}^{+}{ }^{+} \mathrm{T}$ cells. $\mathcal{F}$ Immunol 1997;158:2099-106.

58 Yacyshyn BR, Bowen-Yacyshyn MB, Jewell L, et al. A placebo-controlled Yacyshyn BR, Bowen-Yacyshyn MB, Jewell L, et al. A placebo-controlled
trial of ICAM-1 antisense oligonucleotide in the treatment of Crohn's distrial of ICAM-1 antisense oligonucleotide

59 Panés J, Anderson DC, Miyasaka M, et al. Role of leukocyte-endothelial cell adhesion in radiation-induced microvascular dysfunction in rats. Gastroenterology 1995; 108:1761-9.

60 Kubes P, Jutila M, Payne D. Therapeutic potential of inhibiting leukocyte rolling in ischemia/reperfusion. F Clin Invest 1995;95:2510-19.

61 Kurose I, Pothoulakis C, LaMont JT, et al. Clostridium difficile toxin A-induced microvascular dysfunction. Role of histamine. 7 Clin Invest 1994;94:1919-26. 